\title{
Modeling the dynamics of Lassa fever in Nigeria
}

\author{
Mayowa M. Ojo ${ }^{1 *}$ (D, B. Gbadamosi ${ }^{2}$,Temitope O. Benson ${ }^{3}$, O. Adebimpe ${ }^{4}$ and A. L. Georgina ${ }^{5}$
}

\section{*Correspondence:}

mmojomth@gmail.com

${ }^{1}$ Department of Ecology

and Evolutionary Biology,

University of Kansas,

Lawrence, USA

Full list of author information

is available at the end of the

article

\begin{abstract}
Lassa fever is a zoonotic disease spread by infected rodents known as multimammate rats. The disease has posed a significant and major health challenge in West African countries, including Nigeria. To have a deeper understanding of Lassa fever epidemiology in Nigeria, we present a deterministic dynamical model to study its dynamical transmission behavior in the population. To mimic the disease's biological history, we divide the population into two groups: humans and rodents. We established the quantity known as reproduction number $\mathcal{R}_{0}$. The results show that if $\mathcal{R}_{0}<1$ then the system is stable, otherwise it is unstable. The model fitting was performed using the nonlinear least square method on cumulative reported cases from Nigeria between 2018 and 2020 to obtain the best fit that describes the dynamics of this disease in Nigeria. In addition, sensitivity analysis was performed, and the numerical solution of the system was derived using an iterative scheme, the fifth-order Runge-Kutta method. Using different numeric values for each parameter, we investigate the effect of all highest sensitivity indices' parameters on the population of infected humans and infected rodents. Our findings indicate that any control strategies and methods that reduce rodent populations and the risk of transmission from rodents to humans and rodents would aid in the population's control of Lassa fever.
\end{abstract}

Keywords: Lassa fever, Reproduction number, Stability, Model fitting, Sensitivity analysis, Numerical simulation

\section{Introduction}

The World Health Organization defines disease as any abnormal condition that impairs the function of an organism such as a human, animal, or plant. In humans, diseases are commonly defined as any medical condition characterized by specific symptoms such as pain, distress, dysfunction, or even death. Infectious diseases are caused by microorganisms and can spread from one host to another via direct or indirect transmission [1]. Because of their potential to cause illness and death worldwide, numerous infectious diseases have become a global health challenge. Some of these diseases are unique and are associated with specific regions and environments. Lassa fever (LF) is one of many infectious diseases that are emerging or reappearing in some West African countries. It has caused widespread and serious health problems in West African countries, for instance Nigeria, Liberia, Ghana, Guinea, and Sierra Leone [2]. However, according to the

(c) The Author(s), 2021. Open Access This article is licensed under a Creative Commons Attribution 4.0 International License, which permits use, sharing, adaptation, distribution and reproduction in any medium or format, as long as you give appropriate credit to the original author(s) and the source, provide a link to the Creative Commons licence, and indicate if changes were made. The images or other third party material in this article are included in the article's Creative Commons licence, unless indicated otherwise in a credit line to the material. If material is not included in the article's Creative Commons licence and your intended use is not permitted by statutory regulation or exceeds the permitted use, you will need to obtain permission directly from the copyright holder. To view a copy of this licence, visit http:// creativecommons.org/licenses/by/4.0/. 
Centers for Disease Control and Prevention (CDC), as well as the World Health Organization (WHO), the annual case count for LF ranges between 100,000 and 300,000, with an estimated 5,000 deaths in West Africa [3, 4]. LF was first described in the 1950s, and the viral particle was discovered in 1969 in the northern part of Nigeria, in a city called Lassa in Borno state. Lassa hemorrhagic fever is another name for Lassa fever, and it is contracted through the Lassa virus (LV). The multimammate rat (Mastomys Natalensis) which is of the genus family Arenaviridae is the primary host of LV. The virus is primarily spread to humans through direct contact with contaminated food tainted by the urine or excrement of an infected rodent. Human-to-human transmission is uncommon, but it is possible if a person shares medical equipment with an infected person without proper sterilization. Furthermore, it could be transmitted by dust particles through mucous membranes or skin breaks in humans. Because of its ability to spread from infected animal to human, LF is classified as a zoonotic disease $[3,5]$. In humans, Lassa fever symptoms include headaches, chest pain, nausea, cough, vomiting, diarrhea, muscle pain, abdominal pain, sore throat, and fever. In severe cases, symptoms may include swelling of the face, low blood pressure, fluid in the lungs, and bleeding from the nose, mouth, or vagina. In a more severe case, this disease can result in death within two weeks of the onset of symptoms [6]. Controlling LF in the population can be difficult due to the lack of a vaccine against the virus; however, an antiviral agent known as Ribavirin has been used as a treatment drug in regions where the disease is endemic [3]. According to previous research, the prevalence of Lassa fever is much increased during the wet season because more Mastomys rodents migrate from their natural habitat to the human environment in order to breed and gain proximity. This is the time when human contact with the rodent increases, thus increasing the force of infection and/or the rate of occurrence. Furthermore, previous research has established rainfall as a major ecological factor influencing and contributing to the transmission dynamics of LF, as the transmission probability rate is greater during the rainy season than during the dry season [7]. A variety of socioeconomic factors are also known to play a role in the dynamic spread of Lassa fever. These socioeconomic factors include, to name a few, educational level, occupation, and income, all of which influence the dynamic spread of Lassa fever due to a lack of amenities, malnutrition, an unclean environment, a low standard of living, insufficient health facilities, a lack of a good water source, and personal hygiene [2].

Numerous mathematical modelers and infectious disease experts have conducted studies to further enlighten and provide more information on the transmission dynamics and different approaches to control the endemic disease (see [2-12] for examples). We present a few examples of these studies, along with their methodologies, approaches, and findings. Ifeanyi developed a multiple-patch model in [2] to investigate the effects of socioeconomic class on Lassa fever transmission dynamics. The author performed a sensitivity analysis, which was followed by a numerical illustration of the effect of parameter models for spread of disease and incidence. Their findings show that humans' socioeconomic status has a significant influence on the dynamics of LF transmission. As a result, the study recommends that human socioeconomic classes be considered in order to achieve complete LF eradication in communities where it remains endemic. A study titled "Evaluation of rodent control to fight Lassa fever based on field data and mathematical modelling" was presented 
in [3]. The authors used a mathematical model to experiment various control strategies in rural upper Guinea to determine how long and frequently control should be performed in order to eliminate LF in rural areas. The control strategies employed in this study include annual density control, continuous density control, and rodent vaccination. According to their field data analysis, it is unlikely that a yearly control strategy will reduce LV spillover to humans due to the rapid recovery of the rodent population following rodenticides application. Furthermore, the mathematical model suggests that the best strategy for eradicating LV is continuous control or rodent vaccination. A spatial analysis of Lassa fever data from human cases and infected rodents from 1965 to 2007 was performed in [11], to describe the LF risk maps in West Africa. The authors look into the impact of environmental variables that are extrinsic such as temperature, vegetation, and rainfall on the transmission dynamics of LF in Cameroon. According to the study, rainfall has a strong influence in defining highrisk areas, whereas temperature has a lesser influence in defining high-risk areas. Furthermore, the risk maps revealed that the most dangerous region is situated between Guinea and Cameroon.

Bakare's research [6] developed a non-autonomous system of nonlinear ordinary differential equations that capture the dynamics of LF transmission and seasonal variation in Mastomys rodent birth. The authors of the study evaluate LF disease intervention strategies by predicting optimal intervention best fit in controlling the disease in the population using the elasticity of the equilibria prevalence. Early ribavirin treatments, as well as an early combination of intervention strategies such as effective community hygiene, proper isolation of infected humans, and rodent elimination, will facilitate effective disease control in the population. A mathematical model of the transmission dynamics of Lassa fever infection with control in two different but complementary hosts is presented in [10]. The model includes a death infectious human compartment that can infect a vulnerable individual. According to the study's findings, the best way to control secondary transmission dynamics from human to human is to establish more Lassa fever diagnostic centers and use precautionary burial practices.

Salihu's work is one of the studies that has investigated the dynamics of LF in Nigeria [5]. The authors developed a mechanistic model of the large-scale Lassa fever epidemics in Nigeria from 2016 to 2019 to describe the interaction between human and rodent populations while taking quarantine, isolation, and hospitalization processes into account. Their findings suggest that increasing quarantine and isolation of infected individuals reduce Lassa fever transmission from human to human. Their findings also indicate that across the three outbreaks, initial susceptibility increased from 2016 to 2019. Zhao conducted another study on the large-scale LF outbreak in Nigeria [7]. Their findings suggest that increasing quarantine and isolation of infected individuals reduce Lassa fever transmission from human to human. The authors investigate the epidemiological characteristics of LF epidemics in various Nigeria states by quantifying the relationship between disease reproduction number and local rainfall using the Richards growth model, three-parameter logistic, Gompertz, and Weibull growth models. Surveillance data were also used to fit the respective growth models in order to estimate the reproduction number and epidemic turning points. Overall, the study finds that rainfall has a significant impact on the transmission of LF in Nigeria. 
To better understand the dynamic transmission of Lassa fever in Nigeria, we developed a deterministic model using systems of ordinary differential equations and critically analyzed it both analytically and numerically, in order to provide a more comprehensive understanding of the spread of LF using real cumulative data from the country. The remaining of the article is organized as follows: In Sect. , we present the formulation of the Lassa fever mathematical model. In Sect. , we performed a mathematical analysis on the formulated model, which includes determining the positivity of solutions, the invariant region, and the stability of the Lassa fever free equilibrium. In Sect. , we performed data fitting and parameter estimation. This includes the sensitivity analysis of the model parameters. Numerical results and discussion of the analytical findings and the study conclusion are presented in Sects. and, respectively.

\section{Methods}

\section{Formulation of mathematical model}

The core objectives of this study will be achieved via the development, analysis, parameterization of the model with real data from Nigeria, and simulations with different scenarios of Kermack-McKendrick-type SEIR (susceptible, exposed, infected, and recovered) epidemic model for the transmission dynamics of Lassa fever in Nigeria. Since Lassa fever is a hemorrhagic feverish condition transmitted between two host (humans and rodents), we derived our model by classifying the host population into two, namely human and rodent population.

The total human population at time $t$, denoted by $N_{h}(t)$, is further divided into susceptible, exposed, infectious, and recovered $\left(S_{h}, E_{h}, I_{h}, R_{h}\right)$. Furthermore, the total rodent population at time $t$, denoted by $N_{r}(t)$, is divided into susceptible rodents $\left(S_{r}\right)$ and infectious rodents $\left(I_{r}\right)$. Hence, the total human and rodent population at a given time are given as $N_{h}(t)=S_{h}+E_{h}+I_{h}+R_{h}$ and $N_{r}(t)=S_{r}+I_{r}$, respectively. We model the progression of each subpopulation from one class to another based on their disease status. The susceptible human populace is populated by recruitment rate $\Lambda_{h}$, through birth or immigration and from recovered human individuals due to their loss of immunity at the rate $\tau_{h}$. The susceptible human population is depopulated by infection following effective contact with infected individuals at the rates $\beta_{1}$ given by

$$
\beta_{1}=\frac{\beta_{r} I_{r}+\beta_{h} I_{h}}{N_{h}}
$$

The parameters $\beta_{h}, \beta_{r}$ are the effective transmission probability per contact with infected humans and rodents, respectively. We assume that all human and rodent subpopulation are reduced by natural death at rate $\mu_{h}$ and $\mu_{r}$, respectively. Following the infection of susceptible individuals, they progress to the exposed class. This is the stage where individuals undergo the infection incubation period. Exposed individuals become infectious and progress to increase the infectious class at the rate $\sigma_{h}$. Infectious subpopulation is reduced by recovery due to treatment at the rate $\phi_{h}$ and disease-induced at the rate $\delta_{h}$ (the death due to the disease). The recovered subpopulation is populated by the recovery rate of infectious individuals and further reduced by loss of immunity of recovered individuals. The rodent susceptible subpopulation is populated by birth of rodents at the rate 


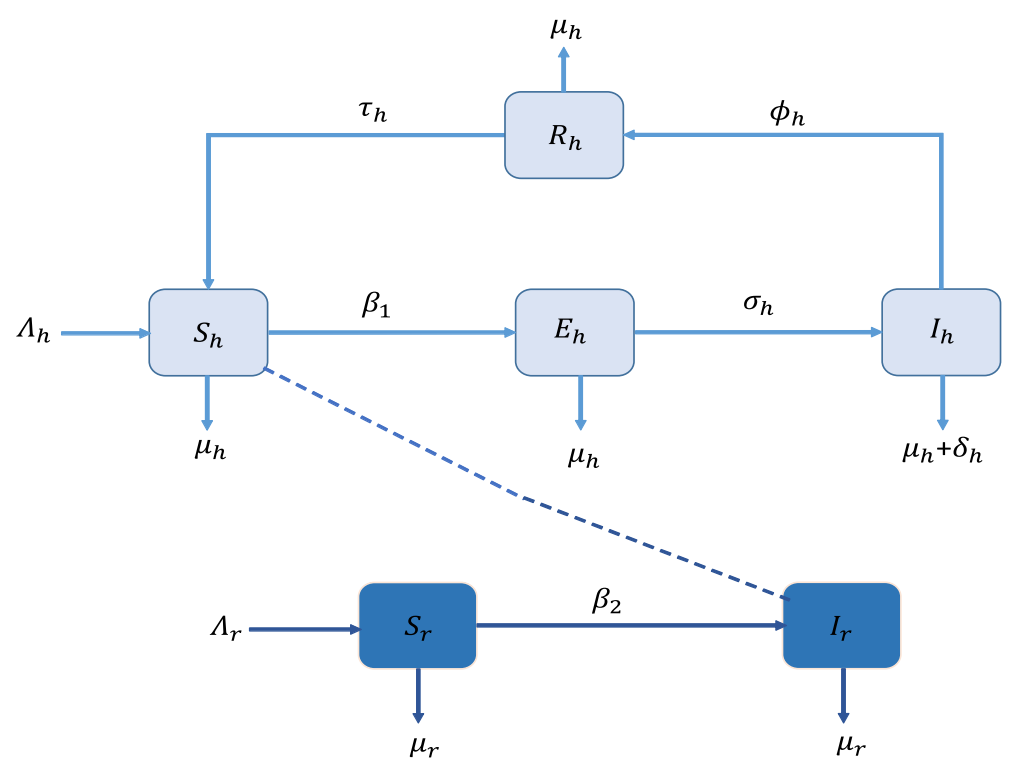

Fig. 1 Schematic diagram of the Lassa fever model (1)

$\Lambda_{r}$. This subpopulation is reduced by infection following effective contact with infected rodents at the rate $\beta_{2}$ given by

$$
\beta_{2}=\frac{\beta_{r} I_{r}}{N_{r}}
$$

The parameter $\beta_{r}$ is the effective transmission probability per contact with infected rodents. Following the descriptions above, the deterministic system of nonlinear differential equations describing the dynamics of Lassa fever in the population is given as

$$
\begin{aligned}
\frac{d S_{h}}{d t} & =\Lambda_{h}+\tau_{h} R_{h}-\beta_{1} S_{h}-\mu_{h} S_{h} \\
\frac{d E_{h}}{d t} & =\beta_{1} S_{h}-\left(\sigma_{h}+\mu_{h}\right) E_{h} \\
\frac{d I_{h}}{d t} & =\sigma_{h} E_{h}-\left(\phi_{h}+\mu_{h}+\delta_{h}\right) I_{h} \\
\frac{d R_{h}}{d t} & =\phi_{h} I_{h}-\left(\mu_{h}+\tau_{h}\right) R_{h} \\
\frac{d S_{r}}{d t} & =\Lambda_{r}-\beta_{2} S_{r}-\mu_{r} S_{r} \\
\frac{d I_{r}}{d t} & =\beta_{2} S_{r}-\mu_{r} I_{r}
\end{aligned}
$$

subject to the following initial conditions $S_{h}(0)>0, E_{h}(0) \geq 0, I_{h}(0) \geq 0, R_{h}(0) \geq 0$, $S_{r}(0)>0$, and $I_{r}(0) \geq 0$. The descriptions of the model parameters and variables are given in Table 1, and the schematic diagram is given in Fig. 1. 
Table 1 Description of the variables and parameters of the Lassa fever model (1)

\begin{tabular}{ll}
\hline Variable & Description \\
\hline$S_{h}$ & Population of susceptible humans \\
$E_{h}$ & Population of exposed humans \\
$I_{h}$ & Population of infectious humans \\
$R_{h}$ & Population of recovered humans \\
$S_{r}$ & Population of susceptible rodents \\
$I_{r}$ & Population of infected rodents \\
\hline Parameter & Description \\
\hline$\Lambda_{h}$ & Recruitment rate of humans through birth or immigration \\
$\tau_{h}$ & Immunity waning rate of humans \\
$\sigma_{h}$ & Disease progression rate of exposed to infected humans \\
$\phi_{h}$ & Recovery rate of infected humans \\
$\mu_{h}$ & Natural mortality rate of humans \\
$\delta_{h}$ & Disease induced death rate of humans \\
$\beta_{h}$ & Transmission probability from humans to humans \\
$\beta_{r}$ & Transmission probability from rodents to humans and rodents \\
$\Lambda_{r}$ & Recruitment rate of rodents through birth \\
$\mu_{r}$ & Natural mortality rate of rodents \\
\hline
\end{tabular}

\section{Mathematical analysis}

\section{Positivity of solutions}

In this section, the basic properties of model (1) will be explored. Since model (1) describes both human and rodents populations during the course of a Lassa fever epidemic, it will only be epidemiologically meaningful if all its state variables are nonnegative for all time $t \geq 0$. In other words, solutions of the model system (1) with nonnegative initial data will remain nonnegative for all time $t>0$.

Lemma 1 The solutions $S_{h}(t), E_{h}(t), I_{h}(t), R_{h}(t), S_{r}(t)$, and $I_{r}$ of the model system (1) with nonnegative initial conditions $S_{h}(0) ; E_{h}(0) ; I_{h}(0) ; R_{h}(0) ; S_{r}(0) ; I_{r}(0)$ will remain nonnegative for all time $t>0$.

Proof $_{t_{1}}=\sup \left\{t>0: S_{h}(t)>0, E_{h}(t)>0, I_{h}(t)>0, R_{h}(t)>0, S_{r}(t)>0, I_{r}(t)>0 \in\right.$ Let $[0, t]\}$

Thus, $t_{1}>0$. It follows from the first equation of system (1), that

$$
\frac{d S_{h}}{d t}=\Lambda_{h}+\tau_{h} R_{h}-\beta_{1} S_{h}-\mu_{h} S_{h} \geq \Lambda_{h}-\beta_{1} S_{h}-\mu_{h} S_{h}
$$

Employing the integrating factor method, this can be written as:

$$
\frac{d}{d t}\left(S_{h}(t) \exp \left[\mu_{h} t+\int_{0}^{t} \beta_{1}(x) d x\right]\right) \geq \Lambda_{h} \exp \left[\mu_{h} t+\int_{0}^{t} \beta_{1}(x) d x\right]
$$

Hence, 


$$
S_{h}\left(t_{1}\right) \exp \left[\mu_{h} t_{1}+\int_{0}^{t_{1}} \beta_{1}(x) d x\right]-S_{h}(0) \geq \int_{0}^{t_{1}} \Lambda_{h}\left(\exp \left[\mu_{h} y+\int_{0}^{y} \beta_{1}(x) d x\right]\right) d y
$$

so that,

$$
\begin{aligned}
S_{h}\left(t_{1}\right) \geq & S_{h}(0) \exp \left[-\mu_{h} t_{1}-\int_{0}^{t_{1}} \beta_{1}(x) d x\right] \\
& +\exp \left[-\mu_{h} t_{1}-\int_{0}^{t_{1}} \beta_{1}(x) d x\right] \times \int_{0}^{t_{1}} \Lambda_{h}\left(\exp \left[\mu_{h} y+\int_{0}^{y} \beta_{1}(x) d x\right]\right) d y>0 .
\end{aligned}
$$

Similarly, it can be shown that $E_{h}(t) \geq 0, I_{h}(t) \geq 0, R_{h}(t) \geq 0, S_{r}(t)>0$, and $I_{r}(t) \geq 0$ for all time $t>0$. Therefore, all the solutions of model (1) remain positive for all nonnegative initial conditions.

\section{Invariant region}

In this section, model (1) will be analyzed in a biologically feasible region as follows. Consider the biologically feasible region consisting of $\Omega=\Omega_{h} \times \Omega_{r} \in \mathcal{R}_{+}^{4} \times \mathcal{R}_{+}^{2}$ with

$$
\Omega_{h}=\left\{S_{h}, E_{h}, I_{h}, R_{h} \in \mathcal{R}_{+}^{4}: N_{h} \leq \frac{\Lambda_{h}}{\mu_{h}}\right\}
$$

and

$$
\Omega_{r}=\left\{S_{r}, I_{r} \in \mathcal{R}_{+}^{2}: N_{r} \leq \frac{\Lambda_{r}}{\mu_{r}}\right\}
$$

It can be shown that the set $\Omega$ is a positively invariant set and global attractor of this system. This implies any phase trajectory initiated anywhere in the nonnegative region $\mathcal{R}_{+}^{6}$ enters the feasible region $\Omega$ and remains in $\Omega$ thereafter.

Lemma 2 The biological feasible region $\Omega=\Omega_{h} \cup \Omega_{r} \subset \mathcal{R}_{+}^{4} \times \mathcal{R}_{+}^{2}$ of the Lassa fever model (1) is positively invariant with nonnegative initial conditions in $\mathcal{R}_{+}^{6}$.

\section{Proof}

The following steps are followed to establish the positive invariance of $\Omega$ (i.e., solutions in $\Omega$ remain in $\Omega$ for all $t>0$ ). The rate of change of the total human and rodent populations $N_{h}$ and $N_{r}$, respectively, are obtained by adding the respective components of model (1) which result to

$$
\begin{aligned}
& \frac{d N_{h}(t)}{d t}=\Lambda_{h}-\mu_{h} N_{h}(t)-\delta_{h} I_{h}(t) \\
& \frac{d N_{r}(t)}{d t}=\Lambda_{r}-\mu_{r} N_{r}(t)
\end{aligned}
$$

so that, 


$$
\frac{d N_{h}(t)}{d t} \leq \Lambda_{h}-\mu_{h} N_{h}(t), \quad \text { and } \quad \frac{d N_{r}(t)}{d t}=\Lambda_{r}-\mu_{r} N_{r}(t)
$$

Hence, $N_{h}(t) \leq N_{h}(0) e^{-\mu_{h} t}+\frac{\Lambda_{h}}{\mu_{h}}\left(1-e^{-\mu_{h} t}\right)$ and $N_{r}(t)=N_{r}(0) e^{-\mu_{r} t}+\frac{\Lambda_{r}}{\mu_{r}}\left(1-e^{-\mu_{r} t}\right)$. In particular, $N_{h}(t) \leq \frac{\Lambda_{h}}{\mu_{h}}$ and $N_{r}(t) \leq \frac{\Lambda_{r}}{\mu_{r}}$ if the total human population and rodent population at the initial instant of time, $N_{h}(0) \leq \frac{\Lambda_{h}}{\mu_{h}}$ and $N_{r}(0) \leq \frac{\Lambda_{r}}{\mu_{r}}$, respectively. So, the region $\Omega$ is positively invariant. Thus, it is consequently adequate to consider the dynamics of Lassa fever governed by model (1) in the biological feasible region $\Omega$, where the model is considered to be epidemiologically and mathematically well posed $[13,14]$.

\section{Existence and Stability of Lassa fever free equilibrium (LFFE)}

The Lassa fever free equilibrium of model (1) denoted by $\mathcal{E}_{0}$ is given by

$$
\mathcal{E}_{0}=\left(S_{h}^{*}, E_{h}^{*}, I_{h}^{*}, R_{h}^{*}, S_{r}^{*}, I_{r}^{*}\right)=\left(\frac{\Lambda_{h}}{\mu_{h}}, 0,0,0, \frac{\Lambda_{r}}{\mu_{r}}, 0\right)
$$

The next-generation matrix method is used on system (1) for determining the reproduction number $\mathcal{R}_{0}$. The epidemiological quantity $\mathcal{R}_{0}$, called the reproduction number, measures the typical number of Lassa fever cases that a Lassa fever-infected individual can generate in a human population that is completely susceptible $[13,15]$. The $\mathcal{R}_{0}$ is used in investigating the local asymptotic stability of the Lassa fever free equilibrium $\mathcal{E}_{0}$. By using the infected compartments $\left(E_{h}^{*}, I_{h}^{*}, I_{r}^{*}\right)$ at the LFFE, and following the notation in $[16,17]$, the Jacobian matrices $F$ and $V$ for the new infection terms and the remaining transfer terms are, respectively, given by

$$
F=\left(\begin{array}{ccc}
0 & \frac{\beta_{h} S_{h}^{*}}{N_{h}^{*}} & \frac{\beta_{r} S_{h}^{*}}{N_{h}^{*}} \\
0 & 0 & 0 \\
0 & 0 & \frac{\beta_{r} S_{h}^{*}}{N_{h}^{*}}
\end{array}\right) \quad \text { and } \quad V=\left(\begin{array}{ccc}
k_{1} & 0 & 0 \\
-\sigma_{h} & k_{2} & 0 \\
0 & 0 & \mu_{r}
\end{array}\right)
$$

It follows that the basic reproduction number of model (1) is given by $\mathcal{R}_{0}=\rho\left(F V^{-1}\right)$, where $\rho$ is the spectral radius of the matrix. Hence,

$$
\mathcal{R}_{0}=\mathcal{R}_{h}+\mathcal{R}_{r}=\frac{\beta_{h} \mu_{r} \sigma_{h}+\beta_{r} k_{1} k_{2}}{k_{1} k_{2} \mu_{r}}
$$

where $\mathcal{R}_{h}=\frac{\beta_{h} \sigma_{h}}{k_{1} k_{2}}, \mathcal{R}_{r}=\frac{\beta_{r}}{\mu_{r}}, k_{1}=\sigma_{h}+\mu_{h}$, and $k_{2}=\mu_{h}+\delta_{h}+\phi_{h}$. From the threshold quantity $\mathcal{R}_{0}$ given above in (5), the quantity $\mathcal{R}_{h}$ measures the contribution of Lassa fever risk caused by human in the population, while the quantity $\mathcal{R}_{r}$ measures the quantity of Lassa fever risk caused by rodent in the population. It must be noted that the increase in any of the threshold quantity will directly upsurge the risk of Lassa fever in the population. The following result is established.

Lemma 3 The Lassa fever free equilibrium $\mathcal{E}_{0}$ of model (1) is locally asymptotically stable in the biological feasible region $\Omega$ if $\mathcal{R}_{0}<1$ and unstable if $\mathcal{R}_{0}>1$. 


\section{Proof}

In order to prove the lemma above, we obtain the Jacobian matrix by evaluating system (1) at Lassa fever free equilibrium $\mathcal{E}_{0}$ as

$$
\mathcal{J}\left(\mathcal{E}_{0}\right)=\left(\begin{array}{cccccc}
-\mu_{h} & 0 & -\beta_{h} & \tau_{h} & 0 & -\beta_{r} \\
0 & -k_{1} & \beta_{h} & 0 & 0 & \beta_{r} \\
0 & \sigma_{h} & -k_{2} & 0 & 0 & 0 \\
0 & 0 & \phi_{h} & -k_{3} & 0 & 0 \\
0 & 0 & -0 & 0 & -\mu_{r} & -\beta_{r} \\
0 & 0 & 0 & 0 & 0 & -\mu_{r}+\beta_{r}
\end{array}\right)
$$

where $k_{1}=\sigma_{h}+\mu_{h}, k_{2}=\mu_{h}+\delta_{h}+\phi_{h}$, and $k_{3}=\mu_{h}+\tau_{h}$. From (6), it is sufficient to show that all the eigenvalues of $\mathcal{J}\left(\mathcal{E}_{0}\right)$ are negative. We obtain the first four eigenvalues as $-\mu_{r},-\mu_{h},-\left(\mu_{r}-\beta_{r}\right)$ and $-k_{3}$. It must be noted that $-\left(\mu_{r}-\beta_{r}\right)$ can also be re-written as $-\mu_{r}\left(1-\mathcal{R}_{r}\right)$, where $\mathcal{R}_{r}=\frac{\beta_{r}}{\mu_{r}}$. The remaining eigenvalues can be obtained from the sub-matrix $\mathcal{M}$ which is written as

$$
\mathcal{M}=\left(\begin{array}{cc}
-k_{1} & \beta_{h} \\
\sigma_{h} & -k_{2}
\end{array}\right)
$$

According to the Routh-Hurwitz condition, all the matrix $\mathcal{M}$ are real and negative if

(i) $\operatorname{Trace}(\mathcal{M})<0$

(ii) $\operatorname{Determinant}(\mathcal{M})>0$

It can be shown that,

$$
\operatorname{Tr}(\mathcal{M})=-\left(k_{1}+k_{2}\right)<0
$$

and

$$
\operatorname{Det}(\mathcal{M})=k_{1} k_{2}-\beta_{h} \sigma_{h}=k_{1} k_{2}\left(1-\frac{\beta_{h} \sigma_{h}}{k_{1} k_{2}}\right)=k_{1} k_{2}\left(1-\mathcal{R}_{h}\right)>0 \quad \text { if } \quad \mathcal{R}_{h} \in \mathcal{R}_{0}<1
$$

Thus, all the eigenvalues of the Jacobian matrix (6) are real and negative if $\left\{\mathcal{R}_{r}, \mathcal{R}_{h}\right\} \in$ $\mathcal{R}_{0}<1$, so that the Lassa fever free equilibrium $\mathcal{E}_{0}$ is locally asymptotically stable and unstable otherwise.

From an epidemiological perspective, Lemma 3 implies that the spread of Lassa fever can be effectively controlled in the population when $\mathcal{R}_{0}$ is less than unity, if the initial sizes of the subpopulations of the model system (1) are in the basin of attraction of the Lassa fever free equilibrium $\mathcal{E}_{0}$.

\section{Existence of Lassa fever endemic equilibrium (EEP)}

We shall investigate the existence of the Lassa fever endemic equilibrium for system (1). The endemic equilibria denoted by $\mathcal{E}_{1}=\left(S_{h}^{* *}, E_{h}^{* *}, I_{h}^{* *}, R_{h}^{* *}, S_{r}^{* *}, I_{r}^{* *}\right)$ represents the steady-state solution in the presence of the disease. By setting the right-hand sides of system (1) to zero and solving simultaneously in terms of the associated force of infection, it gives 


$$
\begin{aligned}
S_{h}^{* *} & =\frac{\Lambda_{h} k_{1} k_{2} k_{3}}{k_{1} k_{2} k_{3} \beta_{1}^{* *}+k_{1} k_{2} k_{3} \mu_{h}-\beta_{1}^{* *} \sigma_{h} \tau_{h} \phi_{h}} \\
E_{h}^{* *} & =\frac{\beta_{1}^{* *} \Lambda_{h} k_{2} k_{3}}{k_{1} k_{2} k_{3} \beta_{1}^{* *}+k_{1} k_{2} k_{3} \mu_{h}-\beta_{1}^{* *} \sigma_{h} \tau_{h} \phi_{h}} \\
I_{h}^{* *} & =\frac{\beta_{1}^{* *} \Lambda_{h} \sigma_{h} k_{3}}{k_{1} k_{2} k_{3} \beta_{1}^{* *}+k_{1} k_{2} k_{3} \mu_{h}-\beta_{1}^{* *} \sigma_{h} \tau_{h} \phi_{h}} \\
R_{h}^{* *} & =\frac{\beta_{1}^{* *} \Lambda_{h} \sigma_{h} \phi_{h}}{k_{1} k_{2} k_{3} \beta_{1}^{* *}+k_{1} k_{2} k_{3} \mu_{h}-\beta_{1}^{* *} \sigma_{h} \tau_{h} \phi_{h}} \\
S_{r}^{* *} & =\frac{\Lambda_{r}}{\beta_{2}^{* *}+\mu_{r}}, \quad I_{r}^{* *}=\frac{\beta_{2}^{* *} \Lambda_{r}}{\mu_{r}\left(\beta_{2}^{* *}+\mu_{r}\right)}
\end{aligned}
$$

where the force of infection is given as

$$
\beta_{1}^{* *}=\frac{\beta_{r} I_{r}^{* *}+\beta_{h} I_{h}^{* *}}{N_{h}^{* *}}, \quad \text { and } \quad \beta_{2}^{* *}=\frac{\beta_{r} I_{r}^{* *}}{N_{r}^{* *}}
$$

Substituting expression (8) into the force of infection (9) at steady state yields the following polynomial

$$
p_{1}\left(\beta_{1}^{* *}\right)^{2}+p_{2} \beta_{1}^{* *}-p_{3}=0
$$

where the coefficients $p_{i}$ for $i=1 \ldots, 3$ of the polynomial are given as

$$
\begin{aligned}
& p_{1}=\mu_{r} \mathcal{R}_{r}\left(\Lambda_{h} k_{2} k_{3}+\Lambda_{h} \sigma_{h} k_{3}+\Lambda_{h} \sigma_{h} k_{3}+\Lambda_{h} \sigma_{h} \phi_{h}\right) \\
& p_{2}=\mu_{r} \mathcal{R}_{r}\left[\Lambda_{h} k_{1} k_{2} k_{3}+\beta_{h} \Lambda_{h} \sigma_{h} k_{3}+\mu_{r}\left(\mathcal{R}_{r}-1\right)\left(k_{1} k_{2} k_{3}-\sigma_{h} \tau_{h} \phi_{h}\right)\right] \\
& p_{3}=\Lambda_{r} k_{1} k_{2} k_{3} \mu_{r}^{2} \mathcal{R}_{r}\left(\mathcal{R}_{r}-1\right)
\end{aligned}
$$

It can be seen that the coefficient $p_{1}$ is positive while the sign of $p_{2}$ and $p_{3}$ depends on the values of the reproduction number. That is, if $\left\{\mathcal{R}_{h}, \mathcal{R}_{r} \in \mathcal{R}_{0}>1\right\}$, then $p_{2}>0$ and $p_{3}>0$. In addition, for $p_{2}$ to be positive, $k_{1} k_{2} k_{3}>\sigma_{h} \tau_{h} \phi_{h}$ so that there is at least one sign change in the sequence of coefficients $p_{1}, p_{2}, p_{3}$. Thus, by Descartes rule of signs, there exists at least one positive real root for (10) whenever $\mathcal{R}_{0}>1$. Therefore, the following result is established.

Lemma 4 The model system (1) has at least one endemic equilibrium whenever $\mathcal{R}_{0}>1$.

\section{Data fitting and parameter estimation}

As provided in Table 2, we obtained our data through three different strategies. Model (1) presented has ten parameters, and realistic values for two of these parameters are available in the literature. We further estimate two demographic parameter values from Nigeria, namely natural death and recruitment rate. The natural death is estimated as $\mu_{h}=\frac{1}{60.45 \times 52}$ per week, where 60.45 years is the average lifespan in Nigeria [18]. Since we assume from model (1) that the total population of human $N_{h}=\frac{\Lambda_{h}}{\mu_{h}}$, substituting the total population of human given as 214, 028, 302 [18], and the estimated value of $\mu_{h}$, we obtain the recruitment rate as 68, 088 per week. We obtained five of the parameters by fitting model (1) to the observed cumulative cases of infected human, based on January 


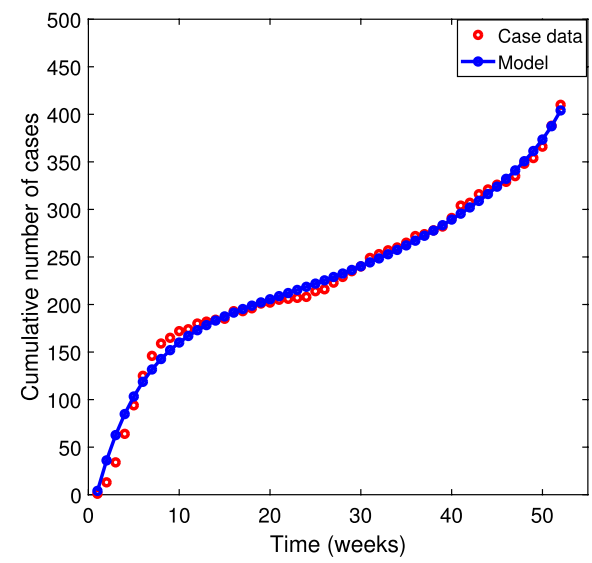

(a)

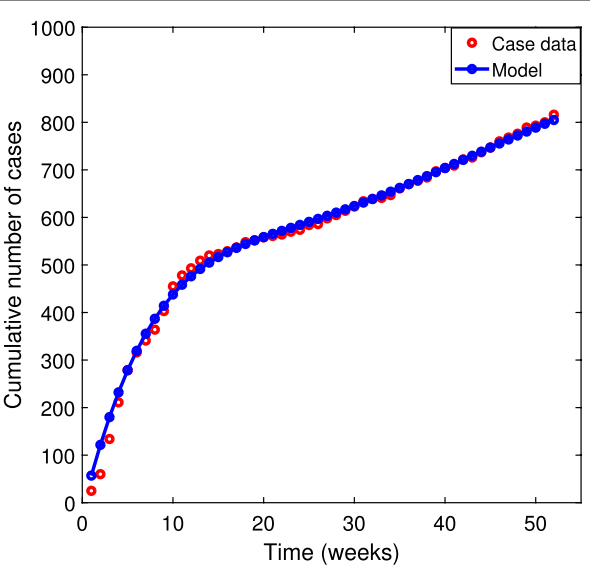

(b)

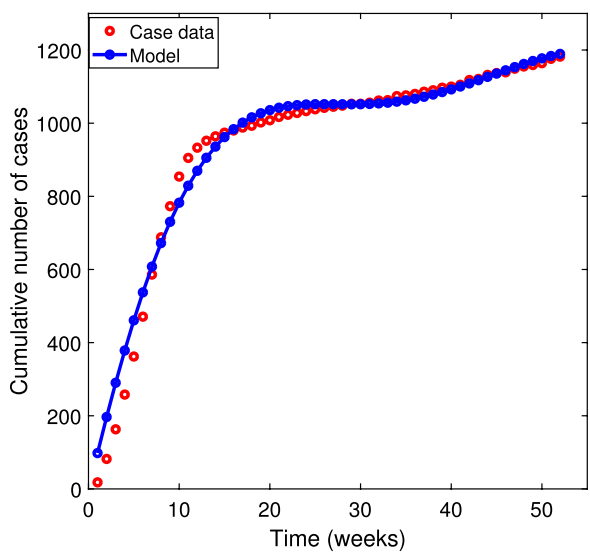

(c)

Fig. 2 Data fitting of the Lassa fever model (1) using a 2018 cumulative cases; b 2019 cumulative cases; c 2020 cumulative cases

2018 to December 2020 Nigerian Lassa fever weekly reported cases. This was obtained from the Nigeria Centre for Disease Control (NCDC) database [19]. Using the mathematical software MATLAB-R2017b, the model fitting was carried out using the nonlinear least square method. The process entails minimizing the sum of the square differences between each observed cumulative confirmed data point and its corresponding confirmed data point from model (1). The root mean square error (RMSE) is sparingly close to zero; this implies that model (1) presented fit very well with the data can be used to make precise predictions for the dynamics of this disease in the populace. All baseline parameter values obtained from fitting the data between 2018 and 2020 are tabulated in Table 2. Furthermore, Fig. 2 depicts the data fitting of the cumulative confirmed cases for 2018, 2019, and 2020, respectively. It must be noted that in Table 2, we presented the estimated mean value of all the parameters; this is defined as the average cumulative cases for the fitted parameter value from 2018 to 2020. The estimated mean value is then used in the next section (except otherwise stated), to carry out sensitivity analysis and to simulate different scenarios of Lassa fever transmission dynamics in Nigeria. 
Table 2 Values of the parameters of the Lassa fever model (1)

\begin{tabular}{llllll}
\hline Parameter & $\mathbf{2 0 1 8}$ & $\mathbf{2 0 1 9}$ & $\mathbf{2 0 2 0}$ & $\begin{array}{l}\text { Estimated mean } \\
\text { value }\end{array}$ & References \\
\hline$\Lambda_{h}$ & 68,088 & 68,088 & 68,088 & 68,088 & Estimated \\
$\tau_{h}$ & 0.3878 & 0.2448 & 1.5736 & 0.7354 & Fitted \\
$\sigma_{h}$ & 0.1873 & 0.1114 & 0.0714 & 0.1234 & Fitted \\
$\phi_{h}$ & 0.0375 & 0.1233 & 0.0236 & 0.0614 & Fitted \\
$\mu_{h}$ & 0.0003 & 0.0003 & 0.0003 & 0.0003 & Estimated \\
$\delta_{h}$ & 0.0024 & 0.0024 & 0.0024 & 0.0024 & {$[20]$} \\
$\beta_{h}$ & 0.0101 & 0.0953 & 0.1479 & 0.0844 & Fitted \\
$\beta_{r}$ & 0.0179 & 0.0627 & 0.0553 & 0.0372 & Fitted \\
$\Lambda_{r}$ & 0.1000 & 0.1000 & 0.1000 & 0.1000 & {$[20]$} \\
$\mu_{r}$ & 0.0627 & 0.0627 & 0.0627 & 0.0627 & Assumed \\
\hline
\end{tabular}

\section{Sensitivity analysis}

In this section, we further explore the impact of each parameters to the transmission dynamics of Lassa fever in Nigeria. To achieve this, we carried out a sensitivity analysis to determine the effect of each threshold quantity parameter, using the given data presented in Table 2 . The sensitivity indices were obtained by employing the approach in $[21,22]$. The sensitivity indices value of each parameters is presented in Table 3, with their respective reproduction number, using the threshold quantity obtained in (5). Furthermore, we present the bar plot of the sensitivity indices in Fig. 3.

Since we employ the use of the estimated mean value parameters as our baseline parameter values in predicting the dynamics of Lassa fever in Nigeria, we discuss the interpretation of the sensitivity indices by using the estimated mean value indices. The result shows that the transmission probability from human to human $\beta_{h}$, and the transmission probability from rodents to humans and rodents $\beta_{r}$ have the highest positive index with the value 0.6887 and 0.3113 , respectively. The positive value implies that decrease (or increase) by $\mathcal{H} \%$ in the transmission probability of infection from humans to humans $\beta_{h}$, or the transmission probability from rodents to humans and rodents $\beta_{r}$ will decrease (or increase) the reproduction number. Likewise, the recovery rate of human $\phi_{h}$, and the natural death rate of rodents $\mu_{r}$ have the highest negative index with the value -0.6591 and -0.3113 , respectively. The negative value infers that increase by $\mathcal{H} \%$ in the recovery rate of human $\phi_{h}$, or the natural death rate of rodents $\mu_{r}$ will decrease the reproduction number by $\mathcal{H} \%$ and vice versa. An epidemiological insight from this result is that any control strategy that reduces the transmission of infection from humans or rodents $\left(\beta_{h}, \beta_{r}\right)$, respectively, and control strategy that increases the recovery rate of human $\phi_{h}$ and the death of rodents $\mu_{r}$ will efficiently shorten the spread of Lassa fever disease in Nigeria. A good example of such control strategy is an effective human hygiene and behavior to reduce the transmission probability of the disease. Also, elimination of infected rodents using any accessible rat killer (such as rodenticides and rat traps), as it is evident that increase in natural death of rodents decreases the reproduction number of the disease. 




(a)

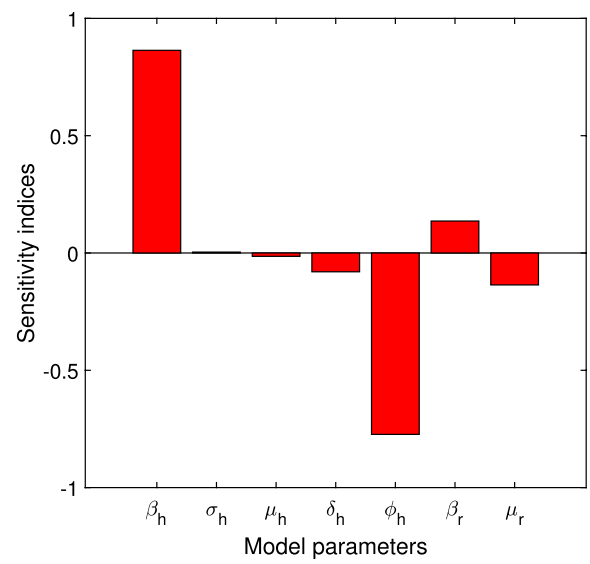

(c)

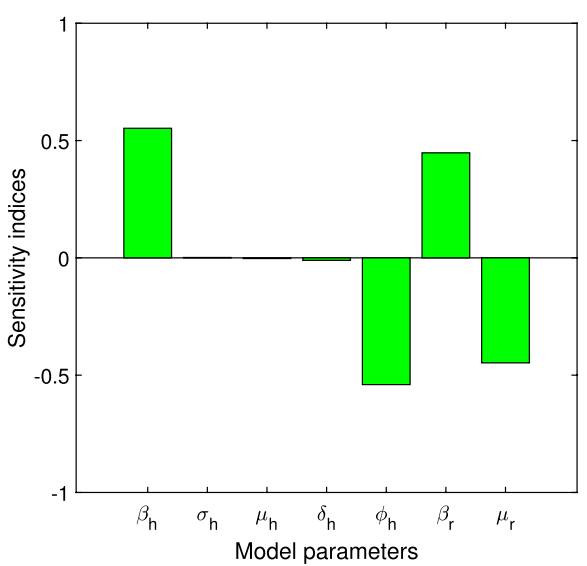

(b)

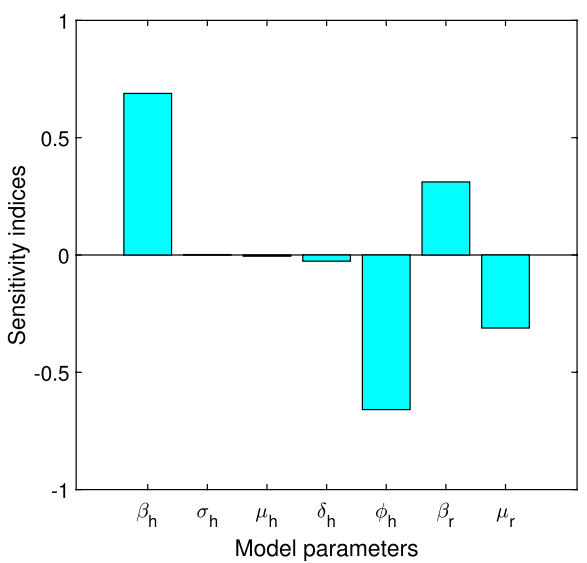

(d)

Fig. 3 Sensitivity indices of the Lassa fever reproduction number $\mathcal{R}_{0}$ with respective to each year parameter value : a 2018 parameter values as given in Table 2; b 2019 parameter values as given in Table 2; c 2020 parameter values as given in Table 2; d Mean parameter values as given in Table 2

Table 3 Sensitivity indices of the reproduction number parameters

\begin{tabular}{llllll}
\hline Parameter & $\mathbf{2 0 1 8}$ & $\mathbf{2 0 1 9}$ & $\mathbf{2 0 2 0}$ & $\begin{array}{l}\text { Estimated mean } \\
\text { value }\end{array}$ & Sign \\
\hline$\beta_{h}$ & 0.4659 & 0.5524 & 0.8638 & 0.6887 & $+v e$ \\
$\beta_{r}$ & 0.5341 & 0.4477 & 0.1362 & 0.3113 & + ve \\
$\sigma_{h}$ & 0.0008 & 0.0016 & 0.0038 & 0.0018 & + ve \\
$\mu_{h}$ & -0.0045 & -0.0029 & -0.0143 & -0.0052 & $-v e$ \\
$\delta_{h}$ & -0.0282 & -0.0107 & -0.0802 & -0.0262 & $-v e$ \\
$\phi_{h}$ & -0.4339 & -0.5402 & -0.7732 & -0.6591 & $-v e$ \\
$\mu_{r}$ & -0.5341 & -0.4477 & 0.1362 & -0.3113 & $-v e$ \\
\hline Threshold quantity & $\mathbf{2 0 1 8}$ & $\mathbf{2 0 1 9}$ & $\mathbf{2 0 2 0}$ & Estimated mean & Sign \\
& & & & value & \\
\hline $\mathcal{R}_{r}$ & 0.2864 & 0.6110 & 0.8819 & 0.5931 & + ve \\
$\mathcal{R}_{h}$ & 0.2499 & 0.7538 & 2.5943 & 1.3120 & + ve \\
$\mathcal{R}_{0}$ & 0.5363 & 1.3648 & 3.4762 & 1.9051 & + ve \\
\hline
\end{tabular}




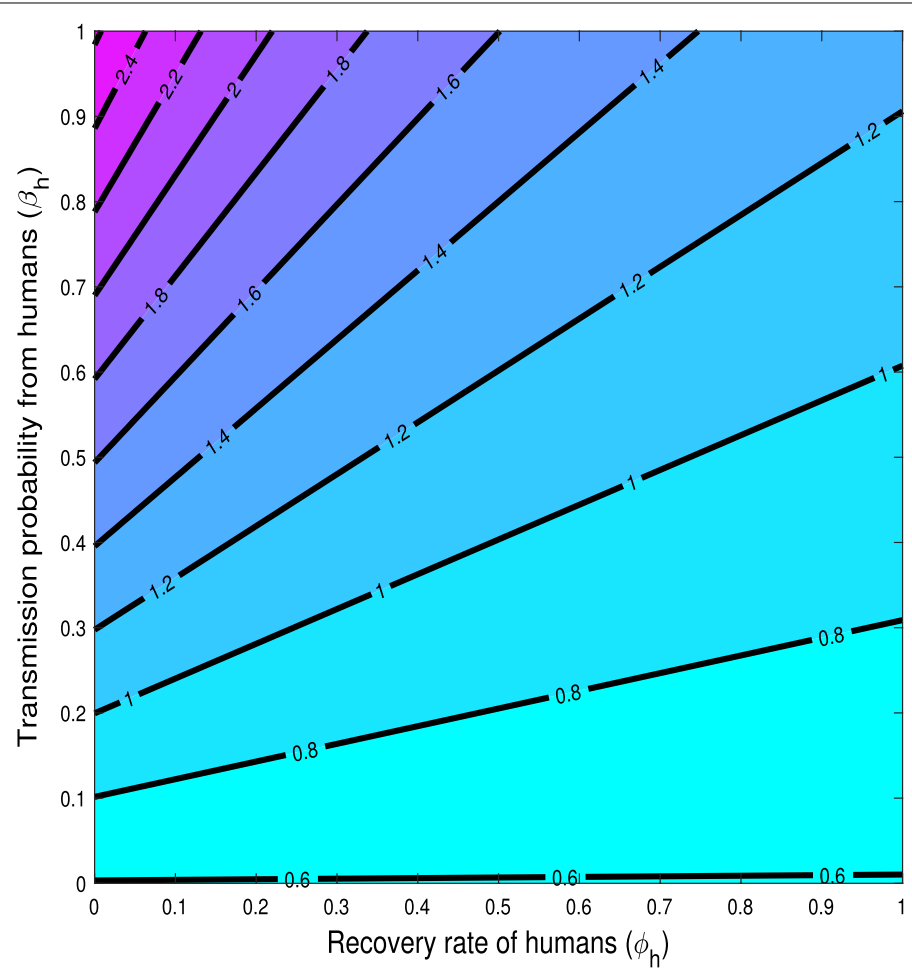

Fig. 4 2-D Contour plot of the reproduction number $\mathcal{R}_{0}$ of Lassa fever model (1), varying recovery rate of humans with respect to transmission probability rate from humans. Parameter values used are as given in Table 2 except for $\delta_{h}=0.4887$ so that $\mathcal{R}_{0}=0.7461<1$.

\section{Results and discussion}

In this section, we present the results of the numerical simulation of our model and its mathematical analysis. These results established our analytical result and findings. We explored the dynamical behavior of infected human and rodent population under different scenarios, using the information from the sensitivity analysis results. We simulated model (1) using MATLAB Solver ode45, which is a six-stage fifth-order Runge-Kutta method. It is imperative to mention that we considered the total infected human population as the sum of both the exposed human and infectious human $\left(E_{h}+I_{h}\right)$. Furthermore, we use the estimated mean value of the parameters given in Table 2 as the baseline parameter value, except otherwise stated.

Figure 4 illustrates a 2-D contour plot which shows the dynamics of the reproduction number, by varying the recovery rate of humans with respect to transmission probability rate from human to human $\beta_{h}$. Increase in the transmission probability rate from human to human increases the reproduction number. For instance, if we fix the recovery rate of humans (x-axis) $\phi_{h}$ to be 0.4 , a transmission probability from human (y-axis) at 0.3 yields a reproduction number between $(1,1.2)$, while when the transmission probability from human is 0.9 , it produces a reproduction number between $(1.6,1.8)$. Furthermore, increase in the recovery rate of humans $\phi_{h}$ reduces the reproduction number. For instance, if we fix the transmission probability from humans (y-axis) $\beta_{h}$ to be 0.5 , a recovery rate of human (y-axis) at 0.4 yields a reproduction number between $(1.2,1.4)$, while when the recovery rate of human is 0.8 , it 


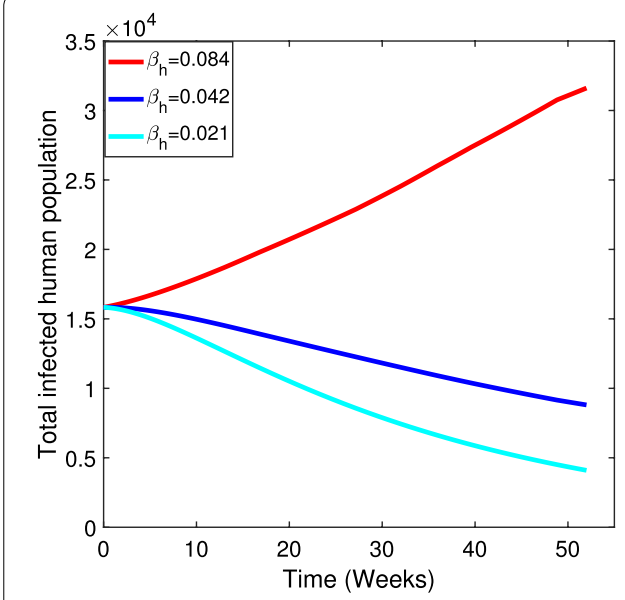

(a)



(b)

Fig. 5 Simulations of model (1) with varying effects of parameters on total infected humans population $\left(E_{h}+I_{h}\right)$ : a transmission probability from humans to humans $\beta_{h}=0.084\left(\mathcal{R}_{0}=1.91\right)$, $\beta_{h}=0.042\left(\mathcal{R}_{0}=1.25\right)$, and $\beta_{h}=0.021\left(\mathcal{R}_{0}=0.92\right)$; b recovery rate of humans $\phi_{h}=0.123\left(\mathcal{R}_{0}=1.91\right)$, $\phi_{h}=0.062\left(\mathcal{R}_{0}=1.90\right), \phi_{h}=0.031\left(\mathcal{R}_{0}=1.89\right) ;$ Other parameter values used are as given in Table 2

produces a reproduction number between $(1,1.2)$. From the results, it can be suggested that to reduce the reproduction number of the disease below unit, a control strategy that facilitates good and speedy recovery rates of humans, together with a reduction of the transmission rate between humans, will be sufficient to curtail the disease in the population.

In Fig. 5, we demonstrate the effects of the transmission probability from human to human $\beta_{h}$ and recovery rate of infected humans $\phi_{h}$, on the infected human population using three different parameter values, respectively. Figure 5 a depicts the effects of $\beta_{h}$ on the dynamics of infected human population. The results show that decrease in transmission rate of the disease from human to human decreases the infected human population. For instance, when $\beta_{h}=0.084$, and $\beta_{h}=0.042$, the reproduction number yields $\mathcal{R}_{0}=1.91$ and $\mathcal{R}_{0}=1.25$, respectively, leaving the disease at her endemic state. However, decreasing $\beta_{h}=0.021$ drives the infected population to her disease-free equilibrium $\mathcal{R}_{0}=0.92<1$. This is the point at which the disease can be curtail in the population, as described in Lemma 3. In Fig. 5b, the result illustrates that an increase in recovery rate of humans increases the total infected human population, given that the transmission probability rate remains at the baseline value. Interestingly, increase in the recovery rate of human is not enough alone to reduce the disease in the population as this can be seen in the estimated reproduction number reported. This can be traced to the effect of the loss of immunity in the recovered individuals. Since the model assumption allows reinfection of recovered humans, when recovery rate increases with a stability of high transmission rate, such dynamics is expected in the population. Hence, it is important to reduce the transmission rate of the disease in order to make the recovery rate of human an effective control strategy to curtail the disease in the population. 


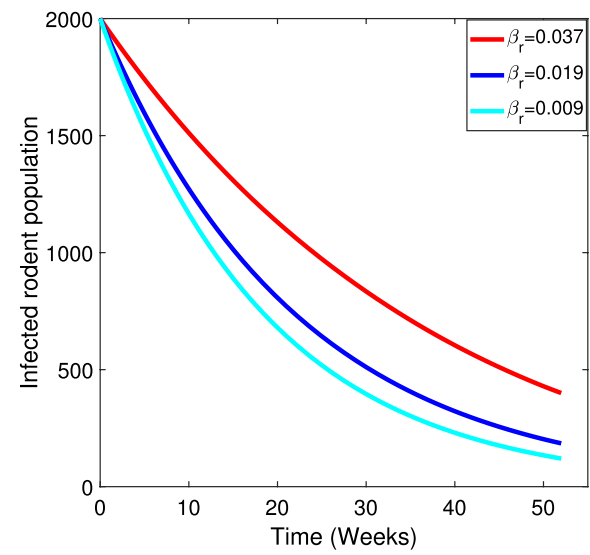

(a)

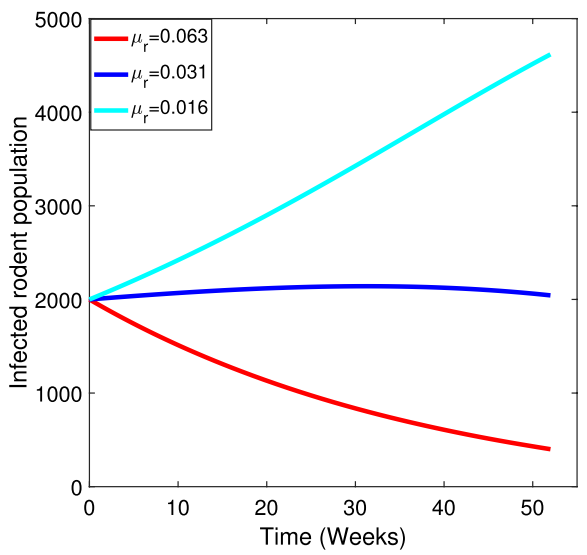

(b)

Fig. 6 Simulations of model (1) with varying effects of parameters on infected rodents population: a transmission probability from rodents to rodents and humans $\beta_{r}=0.037\left(\mathcal{R}_{0}=1.91\right)$,

$\beta_{h}=0.019\left(\mathcal{R}_{0}=1.61\right)$, and $\beta_{r}=0.009\left(\mathcal{R}_{0}=1.46\right)$; b natural death rate of rodents $\mu_{r}=0.063\left(\mathcal{R}_{0}=1.91\right)$, $\mu_{r}=0.031\left(\mathcal{R}_{0}=2.49\right), \mu_{r}=0.016\left(\mathcal{R}_{0}=3.68\right)$. Other parameter values used are as given in Table 2

In order to see the effects of the transmission probability of rodent $\beta_{r}$ and natural death rate of rodent $\mu_{r}$, on the infected rodent population, we simulate the infected rodent population using different parameter values of $\beta_{r}$ and $\mu_{r}$, respectively, in Fig. 6. It can be seen in Fig. 6a that decrease in the transmission probability rate of rodents leads to a decrease in the infected rodent population. For instance, at the baseline parameter value $\beta_{r}=0.037$, the respective reproduction number yields $\mathcal{R}_{0}=1.91$, which draws the final size of the infected rodent population close to 500. However, decreasing the value of $\beta_{r}=0.009$ reduces the reproduction number $\mathcal{R}_{0}=1.46$, which leads to a decrease in infected rodent population. Thus, by limiting the transmission rate of infection between the rodents, the infected rodent population can be reduced to a minimum size. Figure $6 \mathrm{~b}$ illustrates the effect of natural death rate $\mu_{r}$ on the infected rodent population. It was shown that upsurge in natural death rate of the rodents decreases the final size of the infected rodent population. For example, at $\mu_{r}=0.016$, the final infected rodent population size lies between the boundary of $(4000,5000)$, while increase in the natural death of rodent $\left(\mu_{r}=0.063\right)$ reduces the final infected rodent population size below 1000. This result implies that, by reducing the number of rodents in the environment, the infected rodent population can be reduced.

The results in Fig. 7 show the convergence of solution trajectories for the infected humans. This entails using different initial sizes of the population to illustrate the stability behavior of the infected human population size, under little or large perturbation. Figure 7a depicts the stability of the endemic state of the disease when $\mathcal{R}_{0}>1$, while Fig. $7 \mathrm{~b}$ illustrates the stability of the disease-free equilibrium of the model. A simple interpretation of this result is that the infected human population equilibrium will remain the same regardless of any changes in the size of the subpopulation. 


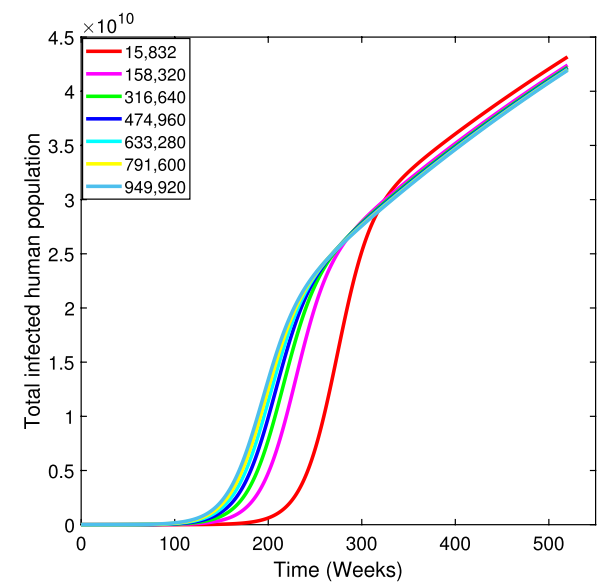

(a)

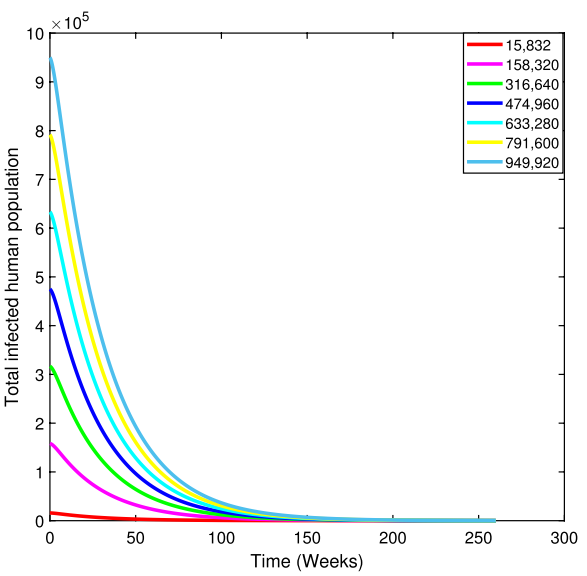

(b)

Fig. 7 Convergence of solution trajectories for infected humans $\left(E_{h}+I_{h}\right)$ with different initial sizes. Parameter values used are as given in Table 2 except for $\mathbf{a} \beta_{h}=1.6889$ so that $\mathcal{R}_{0}=3.22>1 . ; \mathbf{b}$ $\beta_{h}=0.2815, \sigma_{h}=0.3701, \mu_{r}=0.1882, \beta_{r}=0.0124$ so that $\mathcal{R}_{0}=0.50<1$.

\section{Conclusions}

In this study, we formulated a deterministic model using systems of ordinary differential equations to investigate the transmission dynamic of Lassa fever in the population. The population was stratified into human and rodent compartment. The developed model was parameterized by using cumulative reported data obtained from NCDC. Results show that the root mean square error is sparingly close to zero; this implies that the proposed model fit well with the data and can be used to make accurate predictions for the dynamics of this disease in Nigeria. We establish that the LFFE of the model is locally asymptotically stable if the threshold quantity $\mathcal{R}_{0}<1$, and unstable otherwise. We further carried out a sensitivity analysis for the reproduction number to determine the influence of each parameter to the transmission dynamics of Lassa fever in Nigeria. The result proves that the most influential parameters on the reproduction number are the transmission probability rate from human to human $\beta_{h}$, recovery rate of infected humans $\phi_{h}$, transmission probability rate from rodents to humans and rodents $\beta_{r}$, and the natural death rate of rodents $\mu_{r}$. Following this result, numerical simulations were carried out to explore the effect of the most sensitive parameters on the infected human population and rodent population, respectively. Overall, the results from this study suggest that any control strategies that decrease the number of rodent populations, and the transmission probability rate from rodents to humans and rodents, will advance the control of Lassa fever in the population.

Since Lassa fever is endemic in some regions of Africa, it is important to quantify the re-occurrence of this disease outbreak in Nigeria, due to the growth in the reported cases over years. Using the assembled data from 2018 to 2020, we will forecast a future epidemic outbreak using mathematical and computational models. Furthermore, in order to predict the eradication of Lassa fever in Nigeria, we will explore the advantage of multiple control strategies in curtailing this disease in the population. This will be achieved by modifying model (1) with the optimal control problem 
using Pontryagin's maximum principle. In addition to that, since controlling and eradicating any form of diseases in a large population can be both severe and expensive, we will employ the use of a cost-effective analysis to investigate the most cost-effective strategy suitable for use, among various combination of the control strategies.

\section{Abbreviations}

LF: Lassa fever; LV: Lassa virus; LFFE: Lassa fever free equilibrium; NCDC: Nigeria Centre for Disease Control; MATLAB: Matrix Laboratory.

\section{Acknowledgements}

Not applicable.

\section{Authors' contributions}

$B G, T O B, O A, A L G$, and $M M O$ participated in drafting the manuscript. BG and MMO developed the model. MMO analyzed the model and carried out the model fitting and simulations, while TOB and MMO interpreted and discussed the numerical results. All authors read and approved the final manuscript.

\section{Funding}

Not applicable

\section{Availability of data and materials}

All data supporting the findings of this study are included in the list of references and can be obtained at the Nigeria Centre for Disease Control (NCDC) http://www.ncdc.gov.ng/reports.

\section{Declarations}

\section{Competing interests}

The authors declare that they have no competing interests.

\section{Author details}

${ }^{1}$ Department of Ecology and Evolutionary Biology, University of Kansas, Lawrence, USA. ${ }^{2}$ Department of Computer Sciences, Landmark University, Omu-Aran, Kwara State, Nigeria. ${ }^{3}$ Institute for Computational and Data Sciences, University at Buffalo, State University of New York, Albany, USA. ${ }^{4}$ Department of Physical Sciences, Landmark University, Omu-Aran, Kwara State, Nigeria. ${ }^{5}$ Department of Microbiology, Landmark University, Omu-Aran, Kwara State, Nigeria.

Received: 19 February 2021 Accepted: 19 June 2021

Published online: 06 July 2021

\section{References}

1. World Health Organization: Infectious diseases. https://www.who.int/topics/infectiousdiseases/en/. Accessed 9 Feb 2021

2. Onah, I.S., Collins, O.C.: Dynamical system analysis of a Lassa fever model with varying socioeconomic classes. J. Appl. Math. 2020 (2020)

3. Mariën, J., Borremans, B., Kourouma, F., Baforday, J., Rieger, T., Günther, S., Magassouba, N., Leirs, H., Fichet-Calvet, E.: Evaluation of rodent control to fight Lassa fever based on field data and mathematical modelling. Emerg. Microbes Infections 8(1), 640-649 (2019)

4. Olugasa, B.O., Odigie, E.A., Lawani, M., Ojo, J.F., et al.: Development of a time-trend model for analyzing and predicting case-pattern of Lassa fever epidemics in Liberia, 2013-2017. Ann. Afr. Med. 14(2), 89 (2015)

5. Musa, S.S., Zhao, S., Gao, D., Lin, Q., Chowell, G., He, D.: Mechanistic modelling of the large-scale Lassa fever epidemics in Nigeria from 2016 to 2019. J. Theor. Biol. 493, 110209 (2020)

6. Bakare, E., Are, E., Abolarin, O., Osanyinlusi, S., Ngwu, B., Ubaka, O.N.: Mathematical modelling and analysis of transmission dynamics of Lassa fever. J. Appl. Math. 2020 (2020)

7. Zhao, S., Musa, S.S., Fu, H., He, D., Qin, J.: Large-scale Lassa fever outbreaks in Nigeria: quantifying the association between disease reproduction number and local rainfall. Epidemiol Infection 148(2020)

8. Akinpelu, F., Ojo, M.: A mathematical model for the dynamic spread of infection caused by poverty and prostitution in Nigeria. Int. J. Math. Phys. Sci. Res. 4, 33-47 (2016)

9. Akinpelu, F., Ojo, M.: Mathematical analysis of effect of isolation on the transmission of Ebola virus disease in a population. Asian Res. J. Math. 1-12 (2016)

10. Dachollom, S., Madubueze, C.E.: Mathematical model of the transmission dynamics of Lassa fever infection with controls. Math. Model Appl. 5, 65-86 (2020)

11. Fichet-Calvet, E., Rogers, D.J.: Risk maps of Lassa fever in west Africa. PLoS Negl. Trop Dis. 3(3), 388 (2009)

12. Jain, S., Atangana, A.: Analysis of Lassa hemorrhagic fever model with non-local and non-singular fractional derivatives. Int. J. Biomath. 11(08), 1850100 (2018)

13. Lakshmikantham, V., Leela, S., Martynyuk, A.A.: Stability Analysis of Nonlinear Systems. Springer, Berlin (1989)

14. Ojo, M., Akinpelu, F.: Lyapunov functions and global properties of seir epidemic model. Int. J. Chem. Math. Phys. 1(1) (2017) 
15. Oke, S.I., Ojo, M.M., Adeniyi, M.O., Matadi, M.B.: Mathematical modeling of malaria disease with control strategy. Commun. Math. Biol. Neurosci. 2020 (2020)

16. Diekmann, O., Heesterbeek, J.A.P., Metz, J.A.: On the definition and the computation of the basic reproduction ratio $r$ 0 in models for infectious diseases in heterogeneous populations. J. Math. Biol. 28(4), 365-382 (1990)

17. Gbadamosi, B., Ojo, M.M., Oke, S.I., Matadi, M.B.: Qualitative analysis of a dengue fever model. Math. Comput. Appl. 23(3), 33 (2018)

18. Central Intelligence Agency: The world factbook. https://www.cia.gov/the-world-factbook/countries/nigeria/

19. Nigeria Centre for Disease Control: Weekly epidemiological report. https://ncdc.gov.ng/reports/weekly

20. Loyinmi, A.C., Akinfe, K.T., Ojo, A.A.: Qualitative analysis and dynamical behavior of a Lassa Haemorrhagic fever model with exposed rodents and saturated incidence rate (2020)

21. Ojo, M., Gbadamosi, B., Olukayode, A., Oluwaseun, O.R.: Sensitivity analysis of dengue model with saturated incidence rate. Open Access Lib. J. 5(03), 1 (2018)

22. Ojo, M., Akinpelu, F.: Sensitivity analysis of Ebola virus model. Asian Res. J. Math. 1-10 (2017)

\section{Publisher's Note}

Springer Nature remains neutral with regard to jurisdictional claims in published maps and institutional affiliations.

\section{Submit your manuscript to a SpringerOpen ${ }^{\circ}$} journal and benefit from:

- Convenient online submission

- Rigorous peer review

- Open access: articles freely available online

- High visibility within the field

Retaining the copyright to your article

Submit your next manuscript at $>$ springeropen.com 